\title{
ON REARRANGEMENTS OF WALSH-FOURIER SERIES AND HARDY-LITTLEWOOD TYPE MAXIMAL INEQUALITIES ${ }^{1}$
}

\author{
BY WO-SANG YOUNG
}

Communicated by Alberto Calderón, September 22, 1973

ABSTRACT. In this note we study the a.e. convergence properties of certain rearrangements of the Walsh-Fourier series, and maximal functions of the Hardy-Littlewood type that arise from these rearrangements.

The rearrangements are defined as follows. Let $r_{n}$ be the $n$th Rademacher function. For $N=1,2, \cdots$, let $\sigma_{N}$ be a permutation of the nonnegative integers such that $\sigma_{N}(j)=j$ for all $j \geqq N$. If $2^{N} \leqq n<2^{N+1}, n=\sum_{j=0}^{N} \varepsilon_{j} 2^{j}$, where $\varepsilon_{j}=0$ or 1 if $0 \leqq j \leqq N-1$, and $\varepsilon_{N}=1$, we define

We also define $\phi_{0}=1$ and $\phi_{1}=r_{0}$.

$$
\phi_{n}=\prod_{j=0}^{N} r_{\sigma_{N}(j)}^{\varepsilon j} \text {. }
$$

If $\sigma_{N}$ is the identity permutation, $N=1,2, \cdots$, we recover the Walsh system. If $\sigma_{N}(j)=N-j-1,0 \leqq j \leqq N-1,\left\{\phi_{n}\right\}$ is the Walsh-Kaczmarz system. (See [1], [8] and [12].) In general, the system $\left\{\phi_{n}\right\}$ is a rearrangement of the Walsh system within dyadic blocks of indices $2^{N} \leqq n<2^{N+1}$, $N=1,2, \cdots$.

We have the following result on the a.e. convergence of Fourier series with respect to $\left\{\phi_{n}\right\}$. For $f \in L^{1}(0,1)$, let $S_{n} f=\sum_{j=0}^{n-1} \phi_{j} \int_{0}^{1} f \phi_{j} d t$ denote the $n$th partial sum of the Fourier series of $f$ with respect to $\left\{\phi_{n}\right\}$, and $M f=$ $\sup _{n}\left|S_{n} f\right|$.

THEOREM 1. There are absolute constants $C$ and $C_{p}$ such that

(a) $\|M f\|_{p} \leqq C_{p}\|f\|_{p}, f \in L^{p}, 2 \leqq p<\infty$.

(b) $m\{M f>y\} \leqq C \exp \left(-C y /\|f\|_{\infty}\right), y>0, f \in L^{\infty}$.

This implies the a.e. convergence of $S_{n} f$ to $f$ for $f \in L^{p}, 2 \leqq p<\infty$.

If we restrict ourselves to a subclass of rearrangements, we obtain better a.e. convergence results. We say that the permutations $\left\{\sigma_{N}\right\}$ satisfy the

AMS (MOS) subject classifications (1970). Primary 42A56; Secondary 42A20, $46 \mathrm{E} 30$.

${ }^{1}$ This work is part of a doctoral dissertation written under the direction of Professor Richard A. Hunt at Purdue University. 
"block condition" if for each $N=1,2, \cdots, 0 \leqq m \leqq N-1$, there is an integer $k_{N, m}$, with $0 \leqq k_{N, m} \leqq N-m-1$, such that

$$
\left\{\sigma_{N}(0), \cdots, \sigma_{N}(m)\right\}=\left\{k_{N, m}, k_{N, m}+1, \cdots, k_{N, m}+m\right\} .
$$

THEOREM 2. If $\left\{\sigma_{N}\right\}$ satisfies the block condition, then there are absolute constants $C$ and $C_{p}$ such that

(a) $\|m f\|_{p} \leqq C_{p}\|f\|_{p}, f \in L^{p}, 1<p<2$.

(b) $\|M f\|_{1} \leqq C \int_{0}^{1}|f|\left(\log ^{+}|f|\right)^{3} d x+C, f \in L\left(\log ^{+} L\right)^{3}$.

(c) If $\int_{0}^{1}|f|\left(\log ^{+}|f|\right)^{2} \log ^{+} \log ^{+}|f| d x<\infty$, then $S_{n} f$ converges to $f$ a.e.

The absolute constants $C$ and $C_{p}$ in the above theorems are independent of the permutations $\left\{\sigma_{N}\right\}$.

The proofs of these theorems involve a modification of the CarlesonHunt technique (see [3], [6] and [7]), and $L^{p}$ boundedness of certain maximal functions of the Hardy-Littlewood type. We will only give the proofs of the estimates of the maximal functions. Complete proofs of these theorems are contained in [11]. They will appear elsewhere in the Vilenkin group setting in a joint paper with $\mathbf{J}$. Gosselin [5].

To prove Theorem 2, we will show that the maximal operator

$$
f \rightarrow f^{*}=\sup _{0 \leqq m<N ; N} E\left(|f| \mid r_{\sigma_{N}(0)}, \cdots, r_{\sigma_{N}(m)}\right)
$$

is of weak type $(p, p)(p>1)$. Note that for the case where $\sigma_{N}$ is the identity permutation, $N=1,2, \cdots$, this operator is just the usual dyadic HardyLittlewood operator.

LEMMA 1. If $\left\{\sigma_{N}\right\}$ satisfies the block condition, then for $1<p<\infty$,

$$
m\left\{f^{*}>y\right\} \leqq C_{p}^{p} y^{-p} \int_{0}^{1}|f|^{p} d x
$$

where $y>0, f \in L^{p}$, and $C_{p} \leqq p /(p-1)$.

In view of (1), this is a corollary of

LeMma 2. For $1<p<\infty$,

$$
m\left\{\sup _{m, n} E\left(|f| \mid r_{n}, \cdots, r_{n+m}\right)>y\right\} \leqq C_{p}^{p} y^{-p} \int_{0}^{1}|f|^{p} d x,
$$

where $y>0, f \in L^{p}$, and $C_{p} \leqq p /(p-1)$.

Proof. We observe that for any $L^{1}$ function $g$ and integers $n, m \geqq 0$

$$
\begin{aligned}
E\left(g \mid r_{n}, \cdots, r_{n+m}\right) & =E\left(E\left(g \mid r_{0}, \cdots, r_{n+m}\right) \mid r_{n}, \cdots, r_{n+m}\right) \\
& =E\left(E\left(g \mid r_{0}, \cdots, r_{n+m}\right) \mid r_{n}, r_{n+1}, \cdots\right) .
\end{aligned}
$$


The last inequality follows from the independence of the Borel fields $\mathscr{F}\left(r_{0}, \cdots, r_{n+m}\right)$ and $\mathscr{F}\left(r_{n+m+1}, r_{n+m+2}, \cdots\right)$. (See, for example, [4, p. 285].) Therefore

$$
\begin{aligned}
& m\left\{\sup _{m, n} E\left(|f| \mid r_{n}, \cdots, r_{n+m}\right)>y\right\} \\
& \quad \leqq m\left\{\sup _{n} E\left(\sup _{k} E\left(|f| \mid r_{0}, \cdots, r_{k}\right) \mid r_{n}, \dot{r}_{n+1}, \cdots\right)>y\right\} \\
& \leqq y^{-p} \int_{0}^{1} \sup _{k}\left|E\left(|f| \mid r_{0}, \cdots, r_{k}\right)\right|^{p} d x \\
& \leqq C_{p}^{p} y^{-p} \int_{0}^{1}|f|^{p} d x,
\end{aligned}
$$

where $C_{p} \leqq p /(p-1)$. Here we have used Doob's inequality [10, p. 91]. This completes the proof of Lemma 2.

RemARKS. It is interesting to note that the mapping

$$
f \rightarrow \sup _{m, n} E\left(|f| \mid r_{n}, \cdots, r_{n+m}\right)
$$

is not of weak type $(1,1)$. This accounts for the fact that the argument we use only enables us to establish the a.e. convergence result for the rearranged series for functions in the class $L\left(\log ^{+} L\right)^{2} \log ^{+} \log ^{+} L$, whereas, for the Walsh-Fourier series, a similar argument yields the same result for functions in the class $L\left(\log ^{+} L\right) \log ^{+} \log ^{+} L$. (See [9].)

The following is an example of K. H. Moon. We will construct a sequence of functions $\left\{g_{k}\right\}, 0 \leqq g_{k} \in L^{1}$, such that

but

$$
m\left\{\sup _{n, m} E\left(g_{k} \mid r_{n}, \cdots, r_{n+m}\right)>\frac{1}{2}\right\} \geqq \frac{1}{2}, \quad k=1,2, \cdots,
$$

$$
\int_{0}^{1}\left|g_{k}\right| d x \rightarrow 0 \text { as } k \rightarrow \infty .
$$

For each $k=1,2, \cdots, j=0,1, \cdots$, let

$$
A_{k, \jmath}=\left\{r_{k j}=r_{k j+1}=\cdots=r_{k j+k-1}=1\right\} .
$$

Since, for each $k,\left\{A_{k, j}\right\}_{j=0}^{\infty}$ is independent, and

$$
\sum_{j=0}^{\infty} m\left(A_{k, j}\right)=\sum_{j=0}^{\infty} 2^{-k}=\infty,
$$

the Borel-Cantelli Lemma implies that there exists $J_{k}$ such that

$$
m\left(\bigcup_{j=0}^{J_{k}-1} A_{k, j}\right) \geqq \frac{1}{2}
$$


For $k=1,2, \cdots$, define

Thus we have

$$
\begin{aligned}
g_{k}(x) & =2^{k J_{k}} & & \text { if } x \in\left(0,2^{-k-k J_{k}}\right), \\
& =0 & & \text { otherwise. }
\end{aligned}
$$

$$
m\left\{\sup _{m, n} E\left(g_{k} \mid r_{n}, \cdots, r_{n+m}\right)>\frac{1}{2}\right\} \geqq m\left(\bigcup_{j=0}^{J_{k}-1} A_{k, j}\right) \geqq \frac{1}{2},
$$

but

$$
\int_{0}^{1}\left|g_{k}\right| d x=2^{-k} \rightarrow 0 \quad \text { as } k \rightarrow \infty
$$

This shows that $f \rightarrow \sup _{n, m} E\left(|f| \mid r_{n}, \cdots, r_{n+m}\right)$ is not of weak type $(1,1)$.

If we relaxed the block condition on the permutations $\left\{\sigma_{N}\right\}, f \rightarrow f^{*}$ would not be of weak type $(p, p)$ for any $p \geqq 1$. We consider the operator

Let

$$
f \rightarrow \sup _{0 \leqq j<m ; m} E\left(|f| \mid r_{0}, \cdots, r_{j-1}, r_{j+1}, \cdots, r_{m}\right) .
$$

$$
\begin{array}{rlrl}
g_{n}(x)=1 & \text { if } x \in\left(0,2^{-n-1}\right) \\
=0 & & \text { otherwise. }
\end{array}
$$

Then

$$
\begin{array}{rl}
\sup _{0 \leqq j<n} & E\left(g_{n} \mid r_{0}, \cdots, r_{j-1}, r_{j+1}, \cdots, r_{n}\right)(x) \\
= & \frac{1}{2} \text { if } x \in\left(0,2^{-n-1}\right) \cup \bigcup_{j=1}^{n}\left(2^{-j}, 2^{-j}+2^{-n-1}\right), \\
=0 & \text { otherwise. }
\end{array}
$$

Therefore,

$$
m\left\{\sup _{0 \leqq j<m} E\left(g_{n} \mid r_{0}, \cdots, r_{j-1}, r_{j+1}, \cdots, r_{m}\right)>\frac{1}{4}\right\} \geqq(n+1) 2^{-n-1} .
$$

However, $\int_{0}^{1}\left|g_{n}\right|^{p} d x=2^{-n-1}$. This verifies our statement.

To prove Theorem 1 , it is sufficient to have the $L^{p}$ boundedness ( $p \geqq 2$ ) of a weaker operator

$$
f \rightarrow f^{* *}=\sup _{0 \leqq m<N ; N} E\left(\left|f_{N}\right| \mid r_{\sigma_{N}(0)}, \cdots, r_{\sigma_{N}(m)}\right),
$$

where $f_{N}=E\left(f \mid r_{0}, \cdots, r_{N}\right)-E\left(f \mid r_{0}, \cdots, r_{N-1}\right)$. Note that $f^{* *} \leqq f^{*}$.

LEMMA 3. For $2 \leqq p \leqq \infty$,

$$
\left\|f^{* *}\right\|_{p} \leqq 2\|f\|_{p}, \quad f \in L^{p} .
$$


Proof. For $p=2$,

$$
\begin{aligned}
\int_{0}^{1}\left|f^{* *}\right|^{2} d x & \leqq \sum_{N=1}^{\infty} \int_{0}^{1} \sup _{0 \leqq m<N-1}\left|E\left(\left|f_{N}\right| \mid r_{\sigma_{N}(0)}, \cdots, r_{\sigma_{N}(m)}\right)\right|^{2} d x \\
& \leqq 4 \sum_{N=1}^{\infty} \int_{0}^{1}\left|f_{N}\right|^{2} d x=4 \int_{0}^{1}|f|^{2} d x,
\end{aligned}
$$

by Doob's inequality [10, p. 91]. For $p=\infty$,

$$
\left\|f^{* *}\right\|_{\infty} \leqq\left\|f^{*}\right\|_{\infty} \leqq\|f\|_{\infty} .
$$

These norm inequalities together with the Riesz convexity theorem [2] imply our lemma.

\section{REFERENCES}

1. L. A. Balašov, On series with respect to a Walsh system with monotone coefficients, Sibirsk. Mat. Ž. 12 (1971), 25-39=Siberian Math. J. 12 (1971), 18-28. MR 44 \#1982.

2. A. P. Calderón and A. Zygmund, A note on the interpolation of sublinear operators, Amer. J. Math. 78 (1956), 282-288. MR 18, 586.

3. L. Carleson, On the convergence and growth of partial sums of Fourier series, Acta Math. 116 (1966), 135-157. MR 33 \#7774.

4. K. L. Chung, A course in probability theory, Harcourt, Brace and World, New York, 1968. MR 37 \#4842.

5. J. Gosselin and W. S. Young, On rearrangements of Vilenkin-Fourier series which preserve almost everywhere convergence (to appear).

6. R. A. Hunt, On the convergence of Fourier series, Orthogonal Exansions and their Continuous Analogues (Proc. Conf., Edwardsville, Ill., 1967), Southern Illinois Univ. Press, Carbondale, Ill., 1968, pp. 235-255. MR 38 \#6296.

7. - Almost everywhere convergence of Walsh-Fourier series of $L^{2}$ functions, Actes, Congrès Intern. Math. 1970 (2), 655-661.

8. K. H. Moon, Maximal functions related to certain linear operators, Doctoral Dissertation, Purdue University, West Lafayette, Ind., 1972.

9. P. Sjölin, An inequality of Paley and convergence a.e. of Walsh-Fourier series, Ark. Mat. 7 (1969), 551-570. MR 39 \#3222.

10. E. M. Stein, Topics in harmonic analysis related to the Littlewood-Paley theory, Ann. of Math. Studies, no. 63, Princeton Univ. Press, Princeton, N.J.; Univ. of Tokyo Press, Tokyo, 1970. MR 40 \#6176.

11. W. S. Young, Maximal inequalities and almost everywhere convergence, Doctoral Dissertation, Purdue University, West Lafayette, Ind., 1973.

12. - On the a.e. convergence of Walsh-Kaczmarz-Fourier series, Proc. Amer. Math. Soc. (to appear).

Department of Mathematics, Purdue University, West lafayette, Indiana 47907

Current address: Department of Mathematics, Northwestern University, Evanston, Illinois 60201 